\title{
Hunting Season for Primordial Gravitational Waves
}

\author{
The BICEP Array radio telescope, which searches for signatures of \\ gravitational waves in the early Universe, has started an observing run \\ that will break new ground in detection sensitivity.
}

By Matteo Rini

$\mid n$ n 2020, the raging pandemic halted many scientific endeavors around the globe. But after the last flights of the season left the Amundsen-Scott South Pole Station in February, the complex remained "frozen" in a pre-COVID era. There, a few dozen researchers can still mingle without face masks while managing physics, astronomy, and geoscience experiments that run during the six-month-long winter night. The latest experiment to kick off at the South Pole is the BICEP Array telescope, an instrument designed to probe the faint microwave light coming from the infant Universe. After a team assembled the new telescope in the brief austral summer, a lone engineer stayed to tend to the instrument (see Q\&A: Searching for Light in the Darkness of Winter).

Focusing on a small patch of the South Pole's dark winter sky, the BICEP Array will characterize with unprecedented accuracy

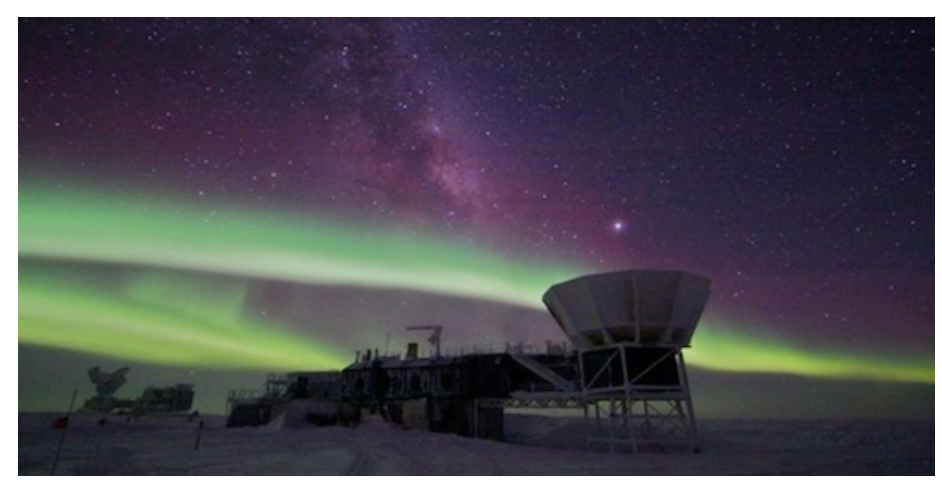

The BICEP Array at the South Pole Station.

Credit: NSF the polarization of the cosmic microwave background (CMB). From these measurements, cosmologists hope to learn about inflation, the Universe's extremely rapid expansion that occurred in the first $10^{-32}$ seconds after the big bang, before a more leisurely expansion began. They will examine subtle polarization patterns, called $B$ modes, that theorists predict were produced by gravitational waves that arose during the inflation epoch.

Few cosmologists doubt that inflation occurred-it explains the Universe's flatness, isotropy, and many other details of cosmic structure. But there isn't an accepted model that explains why and how inflation took place. Measuring $B$ modes, which are direct "messengers" of inflation, could be a crucial step in developing such a model. Much as the 1990s discovery of temperature variations in the CMB allowed theorists to nail down the standard big bang model, BICEP observations may allow researchers, within the next few years, to rule in or rule out some of the most popular models of inflation.

The BICEP Collaboration has been looking for $B$ modes since 2006, using previous versions of their telescope. In March 2014, the collaboration's announcement of a potential discovery sent shockwaves through the scientific community (see Viewpoint: Peering Back to the Beginning of Time). But the excitement didn't last for long: Within a few months, it became clear that galactic dust was the likely explanation for the signal (see Viewpoint: A Clearer View of a Dusty Sky).

The dramatic setback didn't stop the hunt, which is continuing with ever more sensitive instruments and better ways to filter 


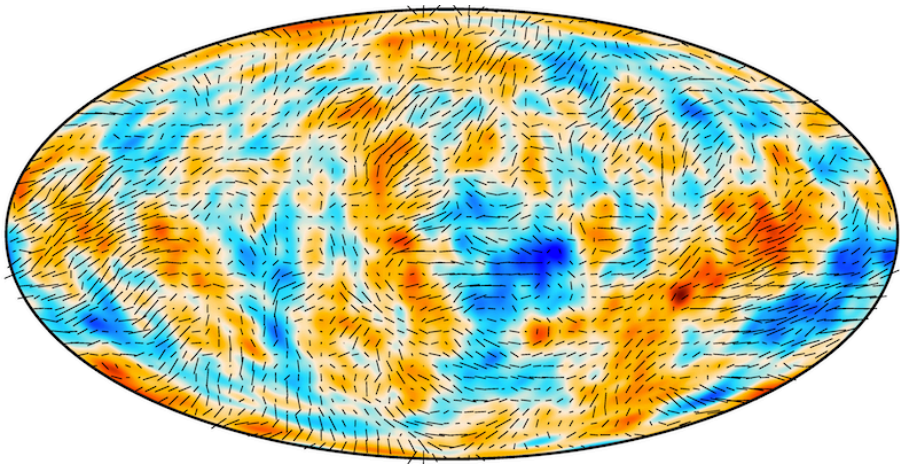

Sky map of CMB polarization measured by the Planck satellite (2018). The segments' directions and lengths represent the polarization direction and amplitude, respectively, and the background represents a coarse-grain map of temperature fluctuations.

Credit: ESA/Planck

out dust and other signal contaminations. At every new data release, BICEP's pointillistic CMB polarization maps get less noisy. "We have been relentlessly observing, and our uncertainties are constantly and predictably shrinking. If there's a signal to be found, it will emerge gradually from the data," says John Kovac of Harvard University, one of the four co-principal investigators (co-PIs) for the BICEP Array.

\section{Clearer Vision of CMB Polarization}

The BICEP Array is the fifth in a series of instrument generations, preceded by BICEP1, BICEP2 (which caught the 2014 signal), the Keck Array (an array of 5 BICEP2-like detectors), and BICEP3. All of these instruments were deployed at the South Pole to take advantage of the clear skies, the high altitude, and one of the driest climates on Earth. With a dry climate, there is little water vapor in the atmosphere that can absorb or emit microwaves. And the telescopes can continuously observe the same patch of sky with the Sun below the horizon for six months straight.

Since flights to and from the South Pole Station are only possible for three months out of each year, the BICEP team went in during the austral summer to reassemble the instrument they had built in Minneapolis. "It was a hell of a lot of work to rebuild the new telescope in just three months. I am really proud of what we accomplished," says Clement Pryke, a BICEP co-PI.

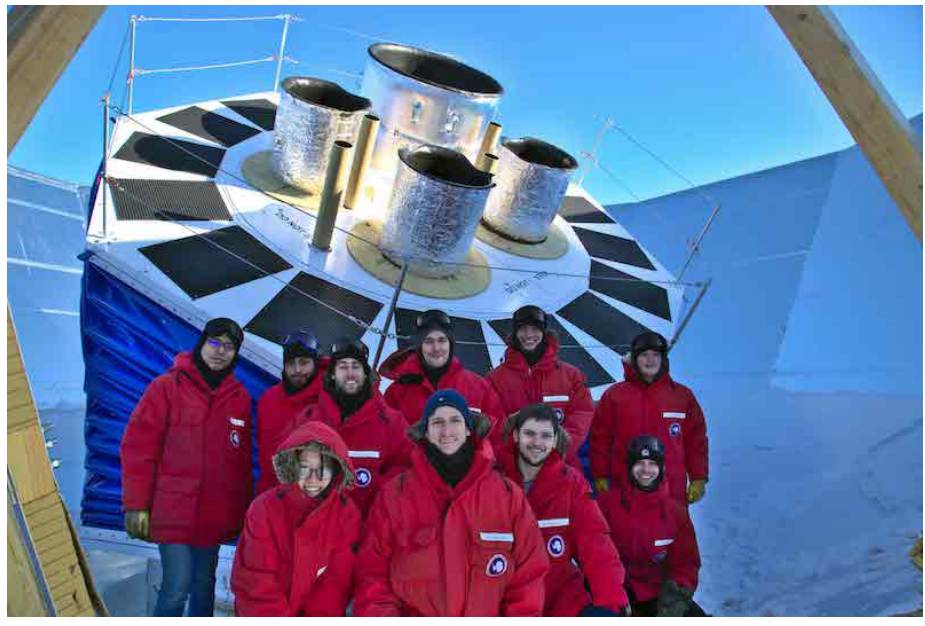

BICEP team after completing the installation of the BICEP Array. The larger receiver is the new BICEP Array receiver, while the smaller receivers are inherited from the Keck Array, a previous generation of the instrument.

Credit: Nathan Precup/University of Minnesota

With the BICEP Array, the instrument has grown in scale and complexity, with dramatic improvements in two main aspects. The first is the number of sensors, or pixels, in the focal plane of the telescope. These sensors are bolometers that detect the polarization signal as minuscule temperature differences. From BICEP1's 98 sensors, the number will rise to about 35,000 for the fully completed BICEP Array. With more sensors, the acquisition of CMB maps gets quicker, boosting sensitivity. A second crucial improvement is the ability to measure different frequencies. While previous BICEP generations focused on one or two frequencies, the Array has sensors at six different frequencies between 30 and $270 \mathrm{GHz}$. This spectral information will be crucial in distinguishing a true gravitational-wave signal from galactic dust and other contaminants.

\section{A Gift from the Universe for Cosmology}

The signal that BICEP will look for is one of many features of the CMB. The CMB's "picture" of the early plasma-filled Universe was taken when electrons and protons in the plasma combined to form neutral hydrogen atoms-making the Universe transparent to light. The spectrum of the CMB peaks at a frequency of $160 \mathrm{GHz}$, corresponding to a temperature of $2.7 \mathrm{~K}$, but small variations in temperature are observed over the sky-evidence of density fluctuations in the primordial plasma. 
The CMB also carries information in its polarization. Much like sunlight bouncing off a surface, CMB radiation became polarized as it scattered from free electrons moving in the primordial plasma. Some electrons moved faster than others, creating variations in the polarization over the sky. "It's an incredible gift from the Universe, which allows us to trace the movement of plasma in the infant Universe," says Kovac.

This connection to the primordial plasma makes CMB polarization a potentially powerful probe of inflation. During the inflationary epoch, the rapid expansion would have produced gravitational waves-spacetime ripples that alternatively compress and expand space. These primordial waves would have modulated the intensity of radiation hitting electrons in the plasma, marking the CMB with the characteristic curling patterns of the $B$ modes. Such patterns, according to many models of inflation, might be detectable by BICEP or other upcoming missions.

\section{Seeing Through the Veil of Dust}

$B$ modes aren't the only polarization pattern in the CMB; there are also $E$ modes. For a given spot in the sky, the $B$ modes curl around a circle centered at the spot, while the $E$ modes are parallel or perpendicular to the radial direction. The two types of modes carry complementary information about the early Universe. The $E$ modes, which are more prominent than the $B$ modes, arise from density fluctuations in the primordial plasma. By contrast, the $B$ modes offer a potential signature of primordial gravitational waves.

The catch is that the $B$ modes can be generated not only by gravitational waves but also by other "foregrounds" produced by astrophysical processes in our Galaxy. Dust in the Milky Way, for example, can emit polarized microwave light with curlicue patterns. Some contamination also comes from the "synchrotron" radiation produced by high-energy electrons flying around in the Galaxy's magnetic fields. The patch of the sky observed by BICEP, which spans an area of 600 square degrees, equal to $1.5 \%$ of the full sky, was selected because previous measurements suggested a low foreground contamination. But until recently, the dust component was poorly understood.

"Dust has been, and still is, the biggest obstacle to a detection," says Raphael Flauger, a theorist with the University of

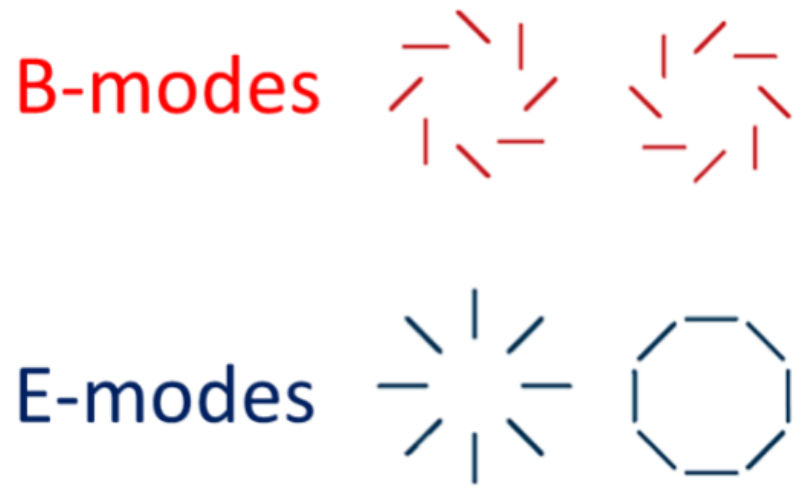

A comparison of the two polarization components in CMB maps. Credit: APS/Michael Schirber

California, San Diego. A cosmologist and a string theorist, he has lately shifted the focus of his research to the impact of foregrounds. "What I really care about is the early Universe, but to get to that information from the CMB we need to remove what hides it," he says.

Foreground removal exploits the frequency dependence of each foreground's contribution. In the frequencies surrounding the CMB peak at $160 \mathrm{GHz}$, dust dominates at the high-frequency end (around $300 \mathrm{GHz}$ ), and synchrotron radiation becomes prominent at the low end (around $30 \mathrm{GHz}$ ). "This leaves a sweet spot around $100 \mathrm{GHz}$, where the foreground is at a minimum," says Pryke. With the BICEP Array's six frequency bands (30, 40, $95,150,220$, and $270 \mathrm{GHz}$ ), the idea is to isolate the dust and synchrotron components through measurements at the highest and lowest frequencies. By cross correlating maps at different frequencies, the foreground contributions at 95 and $150 \mathrm{GHz}$, where the cosmological signal should be largest, can be determined.

This approach, however, only works if the dust contributions at different frequencies are correlated, which means, for example, that the dust signal at $95 \mathrm{GHz}$ can be derived from the dust signal at $270 \mathrm{GHz}$. Flauger's magnetohydrodynamics simulations aim to study exactly that. "The simulation results are reassuring, but that doesn't tell you that nature works in this way," he says. He explains that correlations could be lost if there 

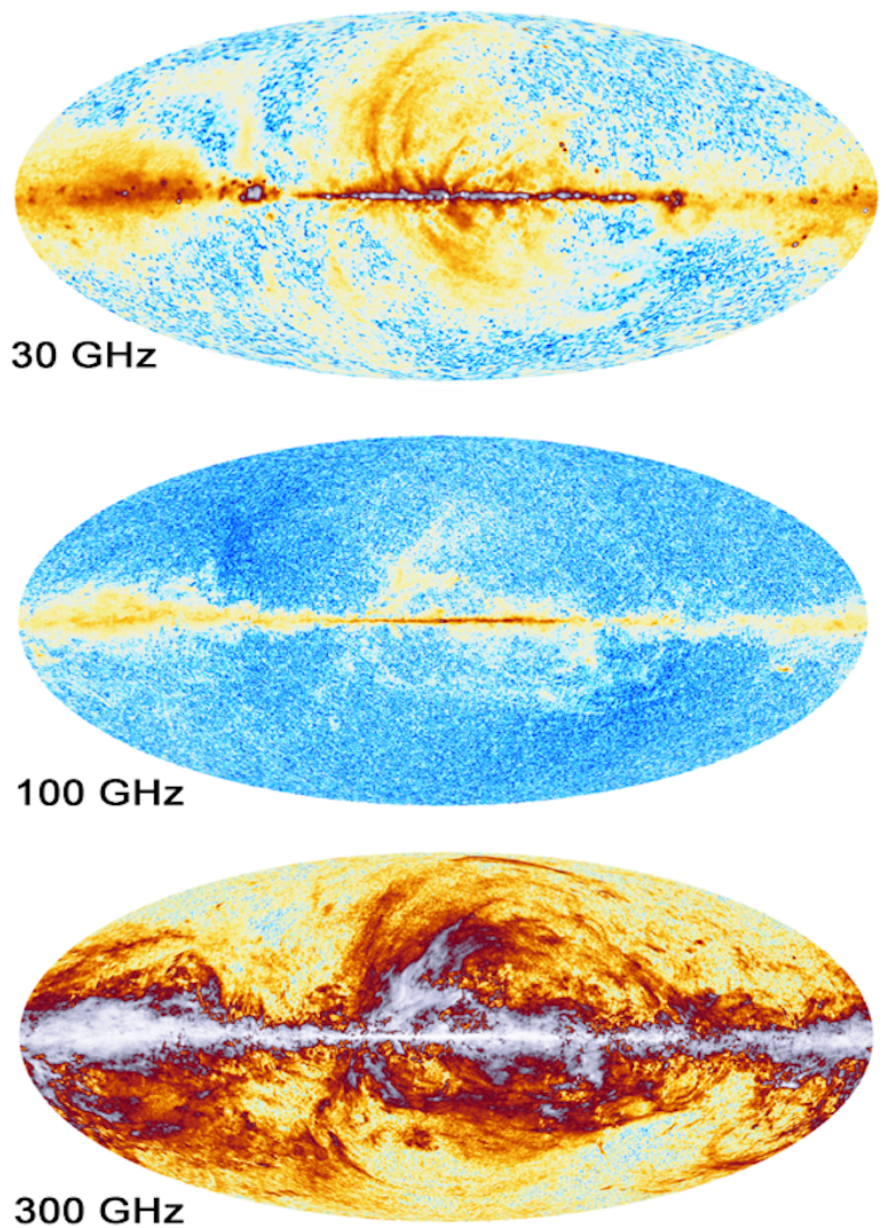

Maps of the polarized CMB sky at different frequencies. At $30 \mathrm{GHz}$, the polarized emission is mostly due to synchrotron emission by free electrons in our Galaxy. At $300 \mathrm{GHz}$, the emission is primarily due to dust. The intermediate frequency range around $100 \mathrm{GHz}$ is a "sweet spot," where the potential signal from primordial gravitational waves should be less contaminated from the foreground emissions.

Credit: ESA/Planck

are different types of dust in different galactic regions, with each type having a different frequency dependence. These issues may be settled with input from the PASIPHAE experiment, which aims to produce 3D maps of galactic dust by measuring the polarization of light from millions of stars, Flauger says.

In addition to galactic foregrounds, gravitational lensing-the distortion of light by gravitational fields-is another effect that could give false signs of primordial gravitational waves. Lensing can cause $E$ mode polarization of the CMB to twist into $B$ modes as the radiation encounters lumps of dark matter. Lensing, however, can be independently measured. The BICEP Array is teaming up with the South Pole Telescope, whose measurements will be used to "de-lens" the CMB maps. "As BICEP's sensitivity gets better and better, accurate delensing becomes essential," says Pryke.

\section{Testing Inflation Models}

By removing dust and lensing, researchers can retrieve the key parameter for inflation studies: the tensor-to-scalar power ratio $r$, which quantifies the strength of the $B$-mode signal compared to the $E$-mode signal. Theory shows that $r$ is related to the energy scale of inflation. The larger the energy associated with inflation's driving force, the larger $r$ would be. Constraints on $r$ have been creeping downward as experiments have gotten more sensitive and as dust has been better understood. The best bounds obtained to date, coming from a joint analysis of data from the Keck Array and from the Planck satellite, limit $r$ to less than 0.06. The BICEP Array Collaboration forecasts that their system will be at least 10 times more sensitive.

The experiments are reaching the sensitivity needed to put important models of inflation to the test. "Some of the simplest and most elegant models of inflation predict signals that should be detectable with the projected sensitivities," says theorist Eva Silverstein of Stanford University. Flauger says that the current limits on $r$ leave room for two well-motivated classes of models, monomial and plateau. (The models are named for the shape of the potential-energy curve that describes how inflation unfolded.) The monomial class predicts $r$ of order of 0.01 , while the plateau class suggests a value of a few times 0.001 . With a projected $r$ sensitivity of a few parts in 1000, testing monomial models "is just around the corner," says Flauger.

\section{More CMB Hunters}

BICEP won't be the only game in town for long. The Simons Observatory, located in the high Atacama Desert of Northern Chile, is set to start measurements in 2021. With a similar number of detectors and six frequency bands between 30 and $280 \mathrm{GHz}$, its sensitivity will be in the same ballpark as that of the BICEP Array. The location, however, implies a different observing strategy. "There are huge tradeoffs in the choice of Chile versus the South Pole," says experimentalist Suzanne 


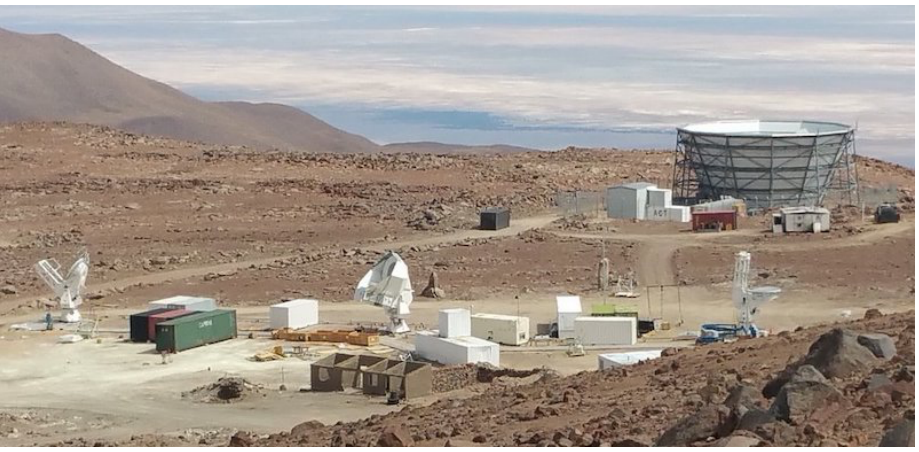

The Simons Observatory will be located at another premier location for cosmological observations-the Atacama Desert in Northern Chile.

Credit: Mark Devlin/University of Pennsylvania

Staggs of Princeton University, spokesperson for the Simons Observatory. BICEP can easily follow a tiny patch of the sky and observe it over and over during the winter night to get a very low-noise map. Simons' maps will be noisier, but they will cover an order-of-magnitude larger portion of sky, so the overall sensitivity to $B$ modes will be similar.

What's more, Simons will observe a different part of the sky with different foreground contamination and will use different strategies for removing the gravitational lensing contribution. "You are always going to question the detection of such an important signal, so you need confirmation from independent, complementary experiments," says Staggs.

In a decade or so, virtually all $B$-mode researchers will team up to work on a project known as CMB-Stage 4 (S4). This next-generation mission will consist of several observatories, located in Chile, at the South Pole, and possibly at other locations in the northern hemisphere. The number of sensors at each observatory will soar to 500,000, and there will be facilities focusing on both small sky slices, like BICEP, and on larger swaths, like Simons. With another tenfold jump in sensitivity beyond that of the BICEP Array, S4 facilities will be able to test plateau inflation models. Similar sensitivity should be reached by LiteBIRD, a small satellite-based observatory planned for launch around 2028. It will measure CMB polarization patterns over the full sky, which is not possible for Earth-bound observatories.

"By the end of these campaigns, researchers will have vetted very-well-motivated models for inflation, but even a nondetection would be very significant," says Silverstein. The absence of a signal would by no means rule out inflation, but it would point theorists to a distinct class of models involving fundamentally different mechanisms and energy scales, she says.

Matteo Rini is the Deputy Editor of Physics. 